\title{
SYNTHESIS AND PHARMACOLOGICAL SCREENING OF CERTAIN IMIDAZOQUINAZOLONE DERIVATIVES
}

Fatma A. Ragab, Hassanein H. Hassanein, Enayat I. Ali and Hanan H. Georgey

Department of Pharmaceutical Chemistry, Faculty of Pharmacy, Cairo University, Egypt

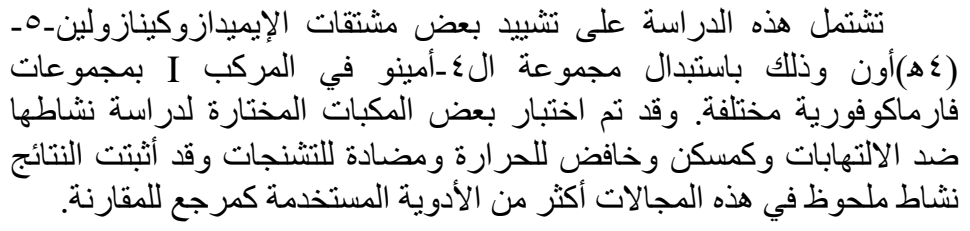

Certain imidazoquinazolin-5(4H)-one derivatives have been synthesized by replacement of the 4-amino group compound I with different moieties of expected biological activity.

Representative example of the synthesized compounds were tested for their anti-inflammatory, analgesic, antipyretic and anticonvulsant activities. Certain derivatives showed activities higher than that of the reference drugs.

\section{INTRODUCTION}

Imidazoquinazolines either linear or angular are well known to exhibit various pharmacological activities, for example cardiovascular ${ }^{1-6}$, bronchodilator $^{7 \& 8}$, antitumor ${ }^{9 \& 10}$, antiinflammatory ${ }^{11-20}$ and anticonvulsant activities $^{21-26}$. In a previous publication 4-amino-1(4-chlorophenyl) imidazo[1,5-a]quinazolin-5(4H)-one I has been synthesized and tested for several pharmacological activities ${ }^{27}$. It showed anti-inflammatory and anticonvulsant activities comparable to that of indomethacin and diazepam respectively which made this compound serves as a useful lead for further design of more active compounds.

The present investigation deals with the synthesis of certain new derivatives of compound $\mathbf{I}$ in which the amino group has been converted by different pharmacophoric groups so as further study the effect of these moieties on the pharmacological potencies. Antiinflammatory, analgesic, antipyretic, and anticonvulsant activities of a number of the synthesized compounds were explored. 


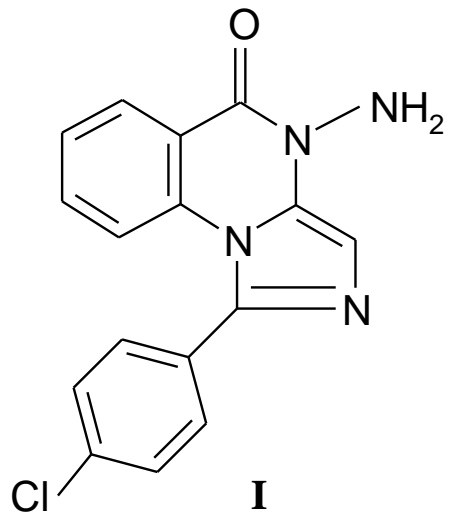

\section{CHEMISTRY}

The key starting compound 4amino-1(4-chlorophenyl)imidazo[1,5a]quinazolin-5(4H)-one I was prepared according to the reported procedure by refluxing methyl 2-[2(4-chlorophenyl)-4-(substituted arylidene)-4,5-dihydro-5-oxoimidazo-1-yl] benzoate with hydrazine hydrate in absolute ethanol ${ }^{27 \& 28}$. The key compound $\mathbf{I}$ was reacted with different aromatic aldehydes, isocyanates or isothiocyanates, acid anhydride and chloroacetyl chloride to give the respected target derivatives II, III, IV and $\mathbf{V}$ respectively, Scheme 1. Compound $\mathbf{V}$ reacted with different secondary amines, potassium salt of substituted aromatic acids, phenytoin sodium or potassium phthalimide to produce the corresponding 4(substituted aminometylcarbonylamino)-1-(4-chlorophenyl)imidazo [1,5-a]quinazolin-5(4H)-ones VI, 1(4-chlorophenyl)-4,5-dihydro-5-oxoimidazo[1,5-a]quinazolin-4-yl-amino- carbonylmethyl benzoates VII. 1-(4chlorophenyl)-4-(4-oxo-5,5-diphenylimidazolin-2-yl)-oxymethylcarbonyl aminoimidazo[1,5-a]quinazolin5(4H)-one VIII and 1-(4chlorophenyl)-4-phthalimidomethyl carbonylamino-imidazo[1,5-a]quinazolin-5(4H)-one IX respectively, Scheme 2. By reacting I with $\mathrm{NaNO}_{2}$ / $\mathrm{HCl}$, neither the hydroxamic acid derivative $\mathbf{X}$ nor the diazonium salt XI were obtained, instead the tetracyclic fused system XIII was obtained. A possible mechanism for the formation of the tetracyclic fused system is illustrated in Scheme 3.

\section{EXPERIMENTAL}

Melting points were carried out by the open capillary tube method using a Gallenkamp digital melting point apparatus and are uncorrected. Microanalyses were carried out at the microanalytical center, Cairo University. Infrared spectra were run on Shimadzu 435 IR spectrophotometer and Bruker Vector 22 FT IR (Fourier Transform Infrared Spectophotometer), and expressed in wave number $\left(\mathrm{cm}^{-1}\right)$, using potassium bromide pellets. Ultraviolet spectra were recorded in absolute ethanol on Shimadzu 265 UV- visible recording spectrophotometer. ${ }^{1} \mathrm{H}-\mathrm{NMR}$ spectra were obtained on Varian Gemini 200, 200MHZ, the chemical shifts were expressed in $\delta \mathrm{ppm}$ units using tetramethylsilane as the internal standard. Mass spectra were 
performed on Hewlett Packard 5988, at $70 \mathrm{e} \mathrm{V.}$

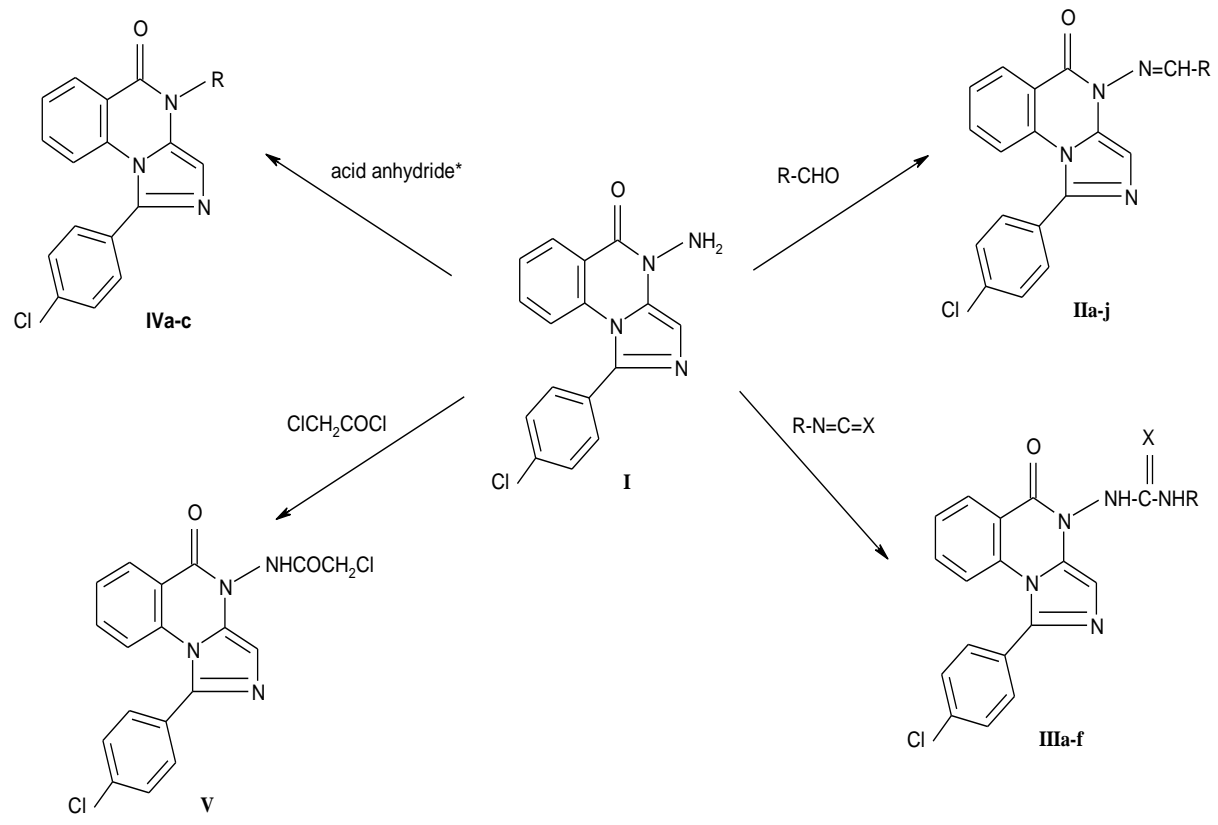

* Acid anhydride = acetic, succinic, or phthalic anhydride.

\section{Scheme 1}




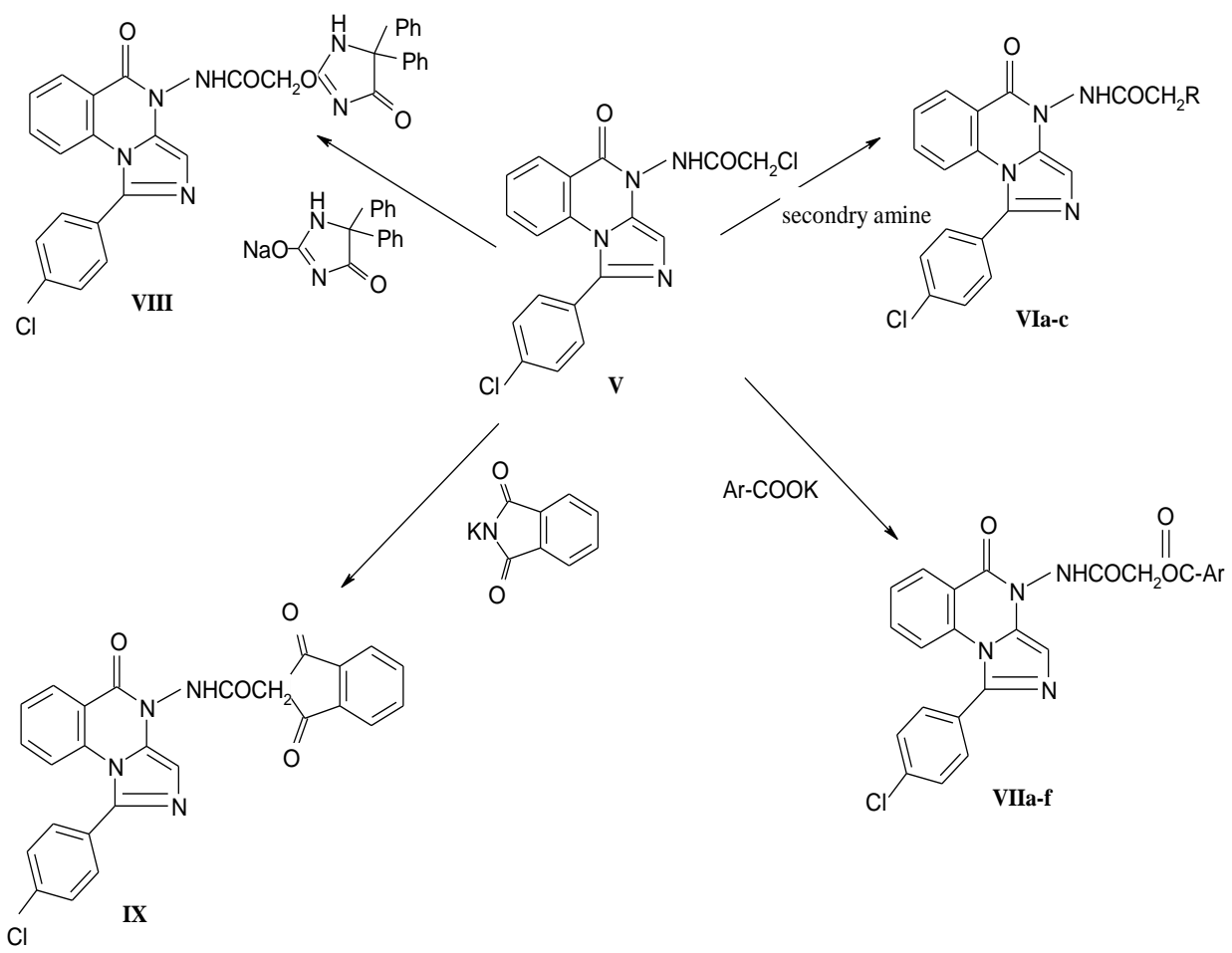

\section{Scheme 2}

Scheme 3

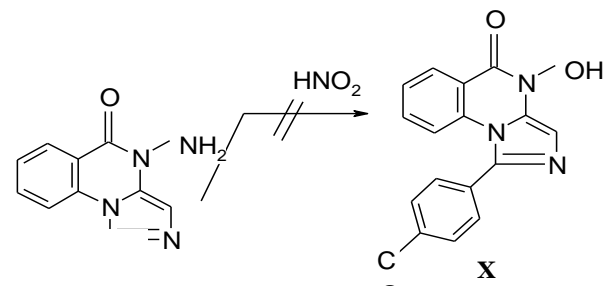

32

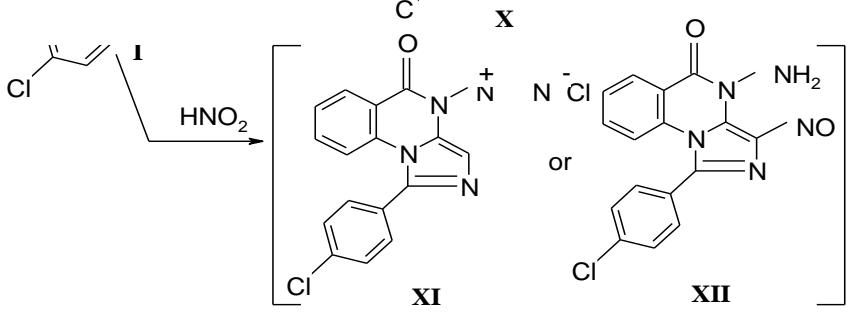


1-(4-Chlorophenyl) 4-(substituted arylidenamino and 3-phenyl-2propenylidenamino) -imidazo $[1,5-$ a] quinazolin-5(4H)-ones IIa-j

A mixture of 4-amino-1-(4chlorophenyl)imidazo[1,5-a]quina-

zolin-5(4H)-one I $(0.01 \mathrm{~mol} ; 3.10 \mathrm{~g})$ and the appropriate aromatic aldehyde $(0.01 \mathrm{~mol})$ in absolute ethanol $(20 \mathrm{ml})$ was refluxed for 8 hours. Then ethanol was removed under reduced pressure and the residue was triturated with ice water. The separated solid was filtered and crystallized from ethanol (Table 1). UV $\left[\lambda_{\text {max. }}(\log \varepsilon)\right]$ of compound IIb: 300 (4.57), 226.4 (4.66). IR $\left(\mathrm{cm}^{-1}\right)$ of compound IIa-IIj: $3050 \quad(\mathrm{CH}$ aromatic), 2950-2850 (CH aliphatic), $1680(\mathrm{C}=\mathrm{O}), 760(\mathrm{C}-\mathrm{Cl}) .{ }^{1} \mathrm{H}$ NMR

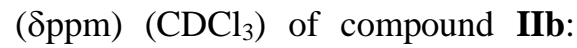
$3.86\left(\mathrm{~s}, 3 \mathrm{H}, \mathrm{OC}_{3}\right), 6.92-7.81(\mathrm{~m}$, $12 \mathrm{H}$, aromatic), 8.35 (d, 1H, $\underline{\mathrm{H}} \mathrm{C}-6)$, 8.91 (s, $1 \mathrm{H}, \mathrm{C} \underline{\mathrm{H}}$ of methine). $\mathrm{MS} \mathrm{m} / \mathrm{z}$ (rel.aband.\%) of compound IIc: 415 (82.73), 339 (38.18), 313 (82.73), 281 (97.27), 237 (50.00), 171 (70.91), 118 (62.72), 60 (100.00).

\section{1-(Alkyl or aryl)-3-[1-(4-chloro- phenyl)- 4,5-dihydro-5-oxo- imidazo[1,5-a]quinazolin-4- yl]carbamide or thiocarbamide (IIIa-IIIf)}

A mixture of 4-amino-1-(4chloro-phenyl)-imidazo[1,5-a]

quinazolin-5(4H)-one I (0. 01 mole; $3.10 \mathrm{~g}$ ), the appropriate aryl or alkyl isocyanate or isothiocyanate $(0.01$ mole) in methylene chloride $(20 \mathrm{ml})$, and triethylamine $(0.5 \mathrm{ml})$ was refluxed for 6 hours. The solid product separated on cooling was filtered, washed with water, and crystallized from benzene (Table 2). UV $\left[\lambda_{\max .}(\log \varepsilon)\right]$ of compound IIIc: 236.0 (4.30), for compound IIIf: 275.2 (4.34). IR $\left(\mathrm{cm}^{-1}\right)$ of compound IIIb: 3300, $3218(2 \mathrm{NH}), 3092(\mathrm{CH}$ aromatic), 2957-2867 (CH aliphatic), $1664(\mathrm{C}=\mathrm{O}), 1620$ (NH bending), 767 (C-Cl). ${ }^{1} \mathrm{H}$ NMR (DMSO-d $\mathrm{d}_{6}$ ) of compound IIIc: $0.96-3.50(\mathrm{~m}, 11 \mathrm{H}$, cyclohexyl), $\quad 5.3 \quad(\mathrm{~s}, \quad 2 \mathrm{H}, \quad 2 \mathrm{NH}$ disappeared by $\left.\mathrm{D}_{2} \mathrm{O}\right), 7.26-7.80(\mathrm{~m}$, $8 \mathrm{H}, \mathrm{C} \underline{\mathrm{H}}$ aromatic), 8.3(d, 1H, $\mathrm{HC}-6)$. ${ }^{1} \mathrm{H} \mathrm{NMR}\left(\mathrm{CDCl}_{3}\right)$ of compound IIIe: $0.8\left(\mathrm{t}, 3 \mathrm{H}, \mathrm{CH}_{2} \mathrm{CH}_{3}\right), 1.25(\mathrm{q}, 2 \mathrm{H}$, $\left.\mathrm{CH}_{2} \mathrm{CH}_{3}\right), \quad 7.26-7.80 \quad(\mathrm{~m}, 8 \mathrm{H}, \quad \mathrm{CH}$ aromatic), 8.3 (d, 1H, $\underline{\mathrm{H}} \mathrm{C}-6)$. MS $\mathrm{m} / \mathrm{z}$ (rel.aband. \%) of compound IIIe: 396.2 (3.17), 339.05 (4.56), 271.95 $\begin{array}{lll}(75.00), & 270.95 \quad(100.00), & 185.05\end{array}$ (9.92), 179 (7.34), 121.75 (16.87), 80.15 (7.14).

\section{1-(4-Chlorophenyl)-4-Substituted} (amido or imido)imidazo [1,5a]quinazolin-5(4H)-ones (IVa-c)

Compound IVa: A mixture of I $(0.01 \mathrm{~mol} ; 3.10 \mathrm{~g})$ and acetic anhydride $(10 \mathrm{ml})$ was heated under reflux for one hour. The reaction mixture was poured onto ice water, filtered, washed with water, and crystallized from aqueous ethanol (Table 1).

Compounds IVb and IVe: A mixture of $\mathbf{I}(0.01 \mathrm{~mol} ; 3.10 \mathrm{~g})$ and the appropriate acid anhydride $(0.01$ $\mathrm{mol})$ in glacial acetic acid $(10 \mathrm{ml})$ was refluxed for 6 hours. Excess 
solvent was distilled under reduced pressure. The residue was triturated with ice water, filtered, washed with water, and crystallized from ethanol (Table 1). UV $\left[\lambda_{\text {max. }}(\log \varepsilon)\right]$ of compound IVa: 384.6 (4.39), 365.0 (4.54), 267.0 (4.18), 245.8 (4.04), 239.4 (4.03). For compound IVb: 236.4 (4.31). IR $\left(\mathrm{cm}^{-1}\right)$ of compound IVa: $3442(\mathrm{NH}), 3068$ (CH aromatic), 2990-2926 (CH aliphatic), 1737, 1700 $(\mathrm{C}=\mathrm{O}), 1620$ (NH bending), 780 (C$\mathrm{Cl})$, for compound IVb: $3100(\mathrm{CH}$ aromatic), 2950-2850 (CH aliphatic), 1730, $1675(\mathrm{C}=\mathrm{O}), 760(\mathrm{C}-\mathrm{Cl}) .{ }^{1} \mathrm{H}$ NMR $\left(\mathrm{CDCl}_{3}\right)$ of compound IVa: 2.30(s, 3H, $\left.\underline{\mathrm{CH}}_{3}\right), 7.4-7.8$ (m, 8H, $\mathrm{CH}$ aromatic), 8.3 (d, 1H, HC-6). MS m/z (rel.aband.\%) of compound IVa: 355 (10.87), 296 (72.25), 271 (100.00), 242 (37.24), 178 (26.83), 139 (22.79), 90 (18.83), 76 (40.23).

\section{4-Chloromethylcarbonylamino-1-} (4-chlorophenyl)-imidazo[1,5-a]quinazolin-5(4H)-oneV

Table 1: Physical and microanalytical data of compounds IIa-j, IVa-c, VIa-d

\begin{tabular}{|c|c|c|c|c|c|c|}
\hline \multirow{2}{*}{ No. } & \multirow{2}{*}{$\mathrm{R}$} & \multirow{2}{*}{ 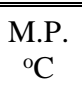 } & \multirow{2}{*}{$\begin{array}{l}\text { Yield } \\
\%\end{array}$} & \multirow{2}{*}{ M.F. M.Wt. } & \multicolumn{2}{|c|}{ Microanalysis } \\
\hline & & & & & Calc. $\%$ & Found $\%$ \\
\hline IIa & & 186 & 75 & $\begin{array}{c}\mathrm{C}_{23} \mathrm{H}_{15} \mathrm{ClN}_{4} \mathrm{O} \\
398.87\end{array}$ & $\begin{array}{cc}\text { C } & 69.25 \\
\text { H } & 3.79 \\
N & 14.04\end{array}$ & $\begin{array}{c}69.10 \\
4.00 \\
14.00\end{array}$ \\
\hline IIb & & 181 & 78 & $\begin{array}{c}\mathrm{C}_{24} \mathrm{H}_{17} \mathrm{ClN}_{4} \mathrm{O}_{2} \\
428.90\end{array}$ & $\begin{array}{ccc}\text { C } & 67.20 \\
H & 3.99 \\
N & 13.06\end{array}$ & $\begin{array}{c}67.50 \\
4.20 \\
13.00\end{array}$ \\
\hline IIc & & 229 & 73 & $\begin{array}{c}\mathrm{C}_{23} \mathrm{H}_{15} \mathrm{ClN}_{4} \mathrm{O}_{2} \\
414.87\end{array}$ & $\begin{array}{cc}\text { C } & 66.58 \\
\text { H } & 3.64 \\
\text { N } & 13.50 \\
\end{array}$ & $\begin{array}{c}66.30 \\
4.00 \\
13.60 \\
\end{array}$ \\
\hline IId & & 201 & 77 & $\begin{array}{c}\mathrm{C}_{24} \mathrm{H}_{17} \mathrm{ClN}_{4} \mathrm{O}_{3} \\
444.90\end{array}$ & $\begin{array}{ccc}\text { C } & 64.78 \\
\text { H } & 3.85 \\
N & 12.59\end{array}$ & $\begin{array}{c}64.60 \\
3.70 \\
12.60\end{array}$ \\
\hline IIe & & 188 & 71 & $\begin{array}{c}\mathrm{C}_{23} \mathrm{H}_{14} \mathrm{ClN}_{5} \mathrm{O}_{3} \\
443.87\end{array}$ & $\begin{array}{cc}\text { C } & 62.23 \\
\text { H } & 3.17 \\
N & 15.77 \\
\end{array}$ & $\begin{array}{c}61.80 \\
3.20 \\
15.70 \\
\end{array}$ \\
\hline
\end{tabular}

A mixture of I $(0.01 \mathrm{~mol} ; 3.10 \mathrm{~g})$ and chloroacetylchloride $(0.01 \mathrm{~mol}$; $1.129 \mathrm{~g} ; 0.79 \mathrm{ml})$, dry benzene (20 $\mathrm{ml})$ and triethylamine $(1 \mathrm{ml})$ was refluxed for 6 hours. The excess solvent was distilled under reduced pressure. The residue was triturated with ice water, filtered, washed with water, and crystallized from ethanol. M.P. $\quad 202^{\circ} \mathrm{C}, \quad$ Yield $\quad 70 \%$. Microanalysis of $\mathrm{C}_{18} \mathrm{H}_{12} \mathrm{Cl}_{2} \mathrm{~N}_{4} \mathrm{O}_{2}$ (387.30) Cacld: C 55.81, H 3.12, N 14.47. Found C 56.10, H 3.40, N 14.90. UV $\left[\lambda_{\max .}(\log \varepsilon)\right]: 237.0$ (4.49). IR $\left(\mathrm{cm}^{-1}\right): 3200(\mathrm{NH}), 3010$ (CH aromatic), 2900-2845 (CH aliphatic), $1714(\mathrm{C}=\mathrm{O}), 1674(\mathrm{NH}$ bending), $\quad 770 \quad(\mathrm{C}-\mathrm{Cl}) . \quad{ }^{1} \mathrm{H} \quad \mathrm{NMR}$ (DMSO-d $)_{6}$ : $4.20\left(\mathrm{~s}, 2 \mathrm{H}, \mathrm{COCH}_{2} \mathrm{Cl}\right.$ ), 7.56-7.96 (m, 8H, CH aromatic), 8.20-8.24 (d, 1H, $\underline{\mathrm{H}} \mathrm{C}-6), 11.6(\mathrm{~s}, 1 \mathrm{H}$, $\mathrm{NH}$ disappeared by $\left.\mathrm{D}_{2} \mathrm{O}\right)$. MS m/z (rel. aband.\%): 386.3 (44.39), 275.2 (42.93), 243.1 (66.83), 178.2 (74.63), 164.0 (22.79), 97.15 (49.27), 71.0 (100.00).

\section{and VIIa-f.}




\begin{tabular}{|c|c|c|c|c|c|c|}
\hline \multirow{2}{*}{ No. } & \multirow{2}{*}{$\mathrm{R}$} & \multirow{2}{*}{$\underset{{ }^{\circ} \mathrm{C}}{\mathrm{M} . \mathrm{P}}$} & \multirow{2}{*}{$\begin{array}{l}\text { Yield } \\
\%\end{array}$} & \multirow{2}{*}{ M.F. M.Wt. } & \multicolumn{2}{|c|}{ Microanalysis } \\
\hline & & & & & Calc. $\%$ & Found $\%$ \\
\hline IIf & & 241 & 69 & $\begin{array}{c}\mathrm{C}_{23} \mathrm{H}_{14} \mathrm{Cl}_{2} \mathrm{~N}_{4} \mathrm{O} \\
433.36\end{array}$ & $\begin{array}{cc}\text { C } & 63.74 \\
\text { H } & 3.25 \\
\text { N } & 12.92\end{array}$ & $\begin{array}{c}63.90 \\
3.20 \\
12.50\end{array}$ \\
\hline IIg & $\mathrm{CH}_{3} \mathrm{O}$ & 204 & 81 & $\begin{array}{c}\mathrm{C}_{25} \mathrm{H}_{19} \mathrm{ClN}_{4} \mathrm{O}_{3} \\
458.92\end{array}$ & $\begin{array}{cc}\text { C } & 65.42 \\
\text { H } & 4.17 \\
\text { N } & 12.20\end{array}$ & $\begin{array}{c}65.80 \\
3.80 \\
12.10\end{array}$ \\
\hline IIh & & 181 & 80 & $\begin{array}{c}\mathrm{C}_{25} \mathrm{H}_{20} \mathrm{ClN}_{5} \mathrm{O} \\
441.94\end{array}$ & $\begin{array}{cc}\mathrm{C} & 67.93 \\
\mathrm{H} & 4.56 \\
\mathrm{~N} & 15.84 \\
\end{array}$ & $\begin{array}{c}68.00 \\
4.10 \\
15.90 \\
\end{array}$ \\
\hline III & & 216 & 72 & $\begin{array}{c}\mathrm{C}_{23} \mathrm{H}_{14} \mathrm{Cl}_{2} \mathrm{~N}_{4} \mathrm{O} \\
433.36\end{array}$ & $\begin{array}{cc}\text { C } & 63.74 \\
\text { H } & 3.25 \\
N & 12.92\end{array}$ & $\begin{array}{c}63.50 \\
3.50 \\
12.50\end{array}$ \\
\hline IIj & $-\mathrm{CH}=\mathrm{C}$ & 179 & 69 & $\begin{array}{c}\mathrm{C}_{25} \mathrm{H}_{17} \mathrm{ClN}_{4} \mathrm{O} \\
424.91\end{array}$ & $\begin{array}{ccc}\text { C } & 70.66 \\
\text { H } & 4.03 \\
N & 13.18\end{array}$ & $\begin{array}{c}71.00 \\
4.30 \\
13.10\end{array}$ \\
\hline IVa & $-\mathrm{NHCOCH}_{3}$ & 153 & 82 & $\begin{array}{c}\mathrm{C}_{18} \mathrm{H}_{13} \mathrm{ClN}_{4} \mathrm{O}_{2} \\
352.80\end{array}$ & $\begin{array}{ccc}\text { C } & 61.27 \\
\text { H } & 3.71 \\
N & 15.87 \\
\end{array}$ & $\begin{array}{c}60.90 \\
4.00 \\
15.50\end{array}$ \\
\hline IVb & & 196 & 78 & $\begin{array}{c}\mathrm{C}_{20} \mathrm{H}_{13} \mathrm{ClN}_{4} \mathrm{O}_{3} \\
392.82\end{array}$ & $\begin{array}{cc}\text { C } & 61.14 \\
\text { H } & 3.33 \\
N & 14.26\end{array}$ & $\begin{array}{c}61.20 \\
3.50 \\
13.90\end{array}$ \\
\hline $\begin{array}{c}\text { Table 1 } \\
\text { IVc }\end{array}$ & $\overline{\text { ntinu }}$ & 256 & 80 & $\begin{array}{c}\mathrm{C}_{24} \mathrm{H}_{13} \mathrm{ClN}_{4} \mathrm{O}_{3} \\
440.86\end{array}$ & $\begin{array}{cc}\text { C } & 65.38 \\
\text { H } & 2.97 \\
N & 12.70\end{array}$ & $\begin{array}{c}65.00 \\
3.30 \\
12.50\end{array}$ \\
\hline VIa & & 206 & 80 & $\begin{array}{c}\mathrm{C}_{20} \mathrm{H}_{18} \mathrm{ClN}_{5} \mathrm{O}_{2} \\
395.87\end{array}$ & $\begin{array}{cc}\text { C } & 60.68 \\
\text { H } & 4.57 \\
\text { N } & 17.69\end{array}$ & $\begin{array}{c}61.00 \\
4.80 \\
18.00\end{array}$ \\
\hline VIb & $-\mathrm{N}^{\prime}$ & 163 & 82 & $\begin{array}{c}\mathrm{C}_{22} \mathrm{H}_{22} \mathrm{ClN}_{5} \mathrm{O}_{2} \\
423.92\end{array}$ & $\begin{array}{c}\text { C } 62.32 \\
\text { H } 5.23 \\
N \quad 16.50\end{array}$ & $\begin{array}{c}62.60 \\
5.40 \\
15.90\end{array}$ \\
\hline VIc & & 206 & 80 & $\begin{array}{c}\mathrm{C}_{20} \mathrm{H}_{18} \mathrm{ClN}_{5} \mathrm{O}_{2} \\
395.87\end{array}$ & $\begin{array}{cc}\text { C } & 60.68 \\
\text { H } & 4.57 \\
\text { N } & 17.69\end{array}$ & $\begin{array}{c}61.00 \\
4.80 \\
18.00\end{array}$ \\
\hline VId & & 164 & 75 & $\begin{array}{c}\mathrm{C}_{22} \mathrm{H}_{20} \mathrm{ClN}_{5} \mathrm{O}_{2} \\
421.91\end{array}$ & $\begin{array}{ccc}\text { C } & 62.62 \\
\text { H } & 4.77 \\
N & 16.59\end{array}$ & $\begin{array}{c}62.40 \\
4.60 \\
16.30\end{array}$ \\
\hline VIIa & & 146 & 81 & $\begin{array}{c}\mathrm{C}_{25} \mathrm{H}_{17} \mathrm{ClN}_{4} \mathrm{O}_{4} \\
472.91\end{array}$ & $\begin{array}{ccc}\text { C } & 63.48 \\
\text { H } & 3.62 \\
N & 11.84 \\
\end{array}$ & $\begin{array}{c}63.40 \\
4.00 \\
12.00\end{array}$ \\
\hline VIIlb & & 142 & 83 & $\begin{array}{c}\mathrm{C}_{25} \mathrm{H}_{17} \mathrm{ClN}_{4} \mathrm{O}_{5} \\
488.91\end{array}$ & $\begin{array}{cc}\text { C } & 61.41 \\
\text { H } & 3.50 \\
N & 11.45 \\
\end{array}$ & $\begin{array}{c}61.80 \\
3.80 \\
11.30 \\
\end{array}$ \\
\hline VIIc & & 228 & 93 & $\begin{array}{c}\mathrm{C}_{25} \mathrm{H}_{16} \mathrm{Cl}_{2} \mathrm{~N}_{4} \mathrm{O}_{4} \\
507.40\end{array}$ & $\begin{array}{cc}\text { C } & 59.17 \\
\text { H } 3.17 \\
\text { N } 11.04 \\
\end{array}$ & $\begin{array}{c}59.50 \\
2.80 \\
11.00\end{array}$ \\
\hline
\end{tabular}




\begin{tabular}{|c|c|c|c|c|c|c|}
\hline \multirow{2}{*}{ No. } & \multirow{2}{*}{$\mathrm{R}$} & \multirow{2}{*}{$\begin{array}{l}\text { M.P. } \\
{ }^{\circ} \mathrm{C}\end{array}$} & \multirow{2}{*}{$\begin{array}{l}\text { Yield } \\
\%\end{array}$} & \multirow{2}{*}{ M.F. M.Wt. } & \multicolumn{2}{|c|}{ Microanalysis } \\
\hline & & & & & Calc. $\%$ & Found $\%$ \\
\hline VIId & & 248 & 94 & $\begin{array}{c}\mathrm{C}_{25} \mathrm{H}_{16} \mathrm{ClN}_{5} \mathrm{O}_{6} \\
517.90\end{array}$ & $\begin{array}{cc}\text { C } & 57.97 \\
\text { H } 3.11 \\
\text { N } 13.52\end{array}$ & $\begin{array}{c}58.20 \\
3.50 \\
13.10\end{array}$ \\
\hline VIIe & & 170 & 71 & $\begin{array}{c}\mathrm{C}_{26} \mathrm{H}_{19} \mathrm{ClN}_{4} \mathrm{O}_{5} \\
502.93\end{array}$ & $\begin{array}{cc}\text { C } & 62.08 \\
\text { H } & 3.80 \\
N & 11.13\end{array}$ & $\begin{array}{c}62.30 \\
4.20 \\
11.10\end{array}$ \\
\hline VIIf & & 124 & 63 & $\begin{array}{c}\mathrm{C}_{25} \mathrm{H}_{16} \mathrm{Cl}_{2} \mathrm{~N}_{4} \mathrm{O}_{4} \\
507.40\end{array}$ & $\begin{array}{cc}\text { C } & 59.17 \\
\text { H } & 3.17 \\
N & 11.04 \\
\end{array}$ & $\begin{array}{c}59.40 \\
3.50 \\
11.10 \\
\end{array}$ \\
\hline
\end{tabular}


Table 2: Physical and microanalytical data of compound IIIa-f.

\begin{tabular}{|c|c|c|c|c|c|c|c|}
\hline \multirow{2}{*}{ No. } & \multirow{2}{*}{$\mathrm{R}$} & \multirow{2}{*}{$X$} & \multirow{2}{*}{$\begin{array}{c}M . P . \\
{ }^{o} C\end{array}$} & \multirow{2}{*}{$\begin{array}{c}\text { Yield } \\
\%\end{array}$} & \multirow{2}{*}{ M.F. M.Wt. } & \multicolumn{2}{|c|}{ Microanalysis } \\
\hline & & & & & & Calc. \% & Found \% \\
\hline IIIa & $-\mathrm{CH}_{2} \mathrm{CH}_{2} \mathrm{CH}_{3}$ & $\mathrm{O}$ & 144 & 89 & $\begin{array}{c}\mathrm{C}_{20} \mathrm{H}_{18} \mathrm{ClN}_{5} \mathrm{O}_{2} \\
395.87\end{array}$ & $\begin{array}{ccc}\text { C } & 60.68 \\
\text { H } & 4.57 \\
N & 17.69 \\
\end{array}$ & $\begin{array}{c}60.40 \\
4.80 \\
17.50 \\
\end{array}$ \\
\hline IIIb & $-\mathrm{CH}_{2} \mathrm{CH}_{2} \mathrm{CH}_{2} \mathrm{CH}_{3}$ & $\mathrm{O}$ & 220 & 67 & $\begin{array}{c}\mathrm{C}_{21} \mathrm{H}_{20} \mathrm{ClN}_{5} \mathrm{O}_{2} \\
409.90\end{array}$ & $\begin{array}{ccc}\text { C } & 61.52 \\
\text { H } & 4.91 \\
\text { N } & 17.08\end{array}$ & $\begin{array}{c}61.20 \\
5.00 \\
17.10\end{array}$ \\
\hline IIIc & & $\mathrm{O}$ & 232 & 90 & $\begin{array}{c}\mathrm{C}_{23} \mathrm{H}_{22} \mathrm{ClN}_{5} \mathrm{O}_{2} \\
435.93\end{array}$ & $\begin{array}{cc}\text { C } & 63.36 \\
H & 5.08 \\
N & 16.06\end{array}$ & $\begin{array}{c}63.70 \\
4.70 \\
15.80\end{array}$ \\
\hline IIId & & $\mathrm{O}$ & 194 & 83 & $\begin{array}{c}\mathrm{C}_{23} \mathrm{H}_{15} \mathrm{Cl}_{2} \mathrm{~N}_{5} \mathrm{O}_{2} \\
464.38\end{array}$ & $\begin{array}{cc}\text { C } & 59.48 \\
\text { H } & 3.25 \\
N & 15.08\end{array}$ & $\begin{array}{c}60.00 \\
3.60 \\
15.10\end{array}$ \\
\hline IIIe & $-\mathrm{CH}_{2} \mathrm{CH}_{3}$ & $S$ & 136 & 93 & $\begin{array}{c}\mathrm{C}_{19} \mathrm{H}_{16} \mathrm{ClN}_{5} \mathrm{OS} \\
397.84\end{array}$ & $\begin{array}{cc}\text { C } & 57.35 \\
\text { H } & 4.05 \\
N & 17.60\end{array}$ & $\begin{array}{c}57.60 \\
4.30 \\
17.70\end{array}$ \\
\hline IIIf & & $S$ & 234 & 80 & $\begin{array}{c}\mathrm{C}_{23} \mathrm{H}_{16} \mathrm{ClN}_{5} \mathrm{OS} \\
445.88\end{array}$ & $\begin{array}{cc}\text { C } & 61.95 \\
\text { H } & 3.61 \\
N & 15.70\end{array}$ & $\begin{array}{c}62.30 \\
4.00 \\
15.80\end{array}$ \\
\hline
\end{tabular}

4-(Substituted aminomethycarbonylamino)-1-(4-chlorophenyl) imidazo[1,5-a]quinaolin-5(4H)-ones VIa-c

A mixture of 4-chloromethylcarbonylamino-1-(4-chlorophenyl)imidazo[1,5-a]quinazolin-5(4H)-one IX $(0.01 \mathrm{~mol} ; 3.87 \mathrm{~g}$.$) and the$ appropriate amine $(0.015 \mathrm{~mol})$ in absolute ethanol $(20 \mathrm{ml})$ was refluxed for 12 hours. The resulting solution was distilled under reduced pressure. The residue was triturated with ice water. The separated solid was filtered, washed with water and crystallized from ethanol (Table 1). UV $\left[\lambda_{\text {max. }}(\log \varepsilon)\right]$ of compound VIc: 277.2 (4.23), 230.0 (4.60). IR $\left(\mathrm{cm}^{-1}\right)$ of compound VIc: $3211(\mathrm{NH}), 3067$ (CH aromatic), 2981-2830 (CH aliphatic), 1720, $(\mathrm{C}=\mathrm{O}), 1692(\mathrm{NH}$ bending), $780 \quad(\mathrm{C}-\mathrm{Cl}) .{ }^{1} \mathrm{H} \quad$ NMR
$\left(\mathrm{CDCl}_{3}\right)$ of compound VIb: 0.94-1.01 (t, $6 \mathrm{H}, 2 \times \mathrm{CH}_{2} \mathrm{CH}_{3}$ ), 2.44-2.60 (q, $\left.4 \mathrm{H}, 2 \times \mathrm{CH}_{2} \mathrm{CH}_{3}\right), 3.29(\mathrm{~s}, 2 \mathrm{H}$, $\left.\mathrm{COCH}_{2}-\right), \quad 7.3-7.8 \quad(\mathrm{~m}, \quad 8 \mathrm{H}, \quad \mathrm{CH}$ aromatic), 8.29 (d, 1H, HC-6). MS $\mathrm{m} / \mathrm{z}$ (rel. aband.\%): of compound VIc: 438 (7.35), 368 (8.53), 298 (9.12), 257 (22.35), 197 (14.12), 158 (30.00), 100 (100.00).

\section{1-(4-Chlorophenyl)-4,5-dihydro-5-} oxo-imidazo[1,5-a] quinazolin -4aminocarbonyl methyl benzoates VIIa-f

A mixture of 4-chloromethylcarbonylamino-1-(4-chlorophenyl)imidazo[1,5-a]quinazolin-5(4H)-one V $\quad(0.01 \mathrm{~mol} ; 3.87 \mathrm{~g})$ and the appropriate substituted potassium benzoate $(0.01 \mathrm{~mol})$ in dimethylformamide $(5 \mathrm{ml})$ was heated in a boiling water bath for 3 hours. The 
resulting solution was cooled, poured on ice water. The solid product was filtered, washed with water, and crystallized from ethanol (Table 1). UV $\left[\lambda_{\max }(\log \varepsilon)\right]$ of compound VIb: 3062. (4.07), 240.2 (4.50), 223.6 (4.60). IR $\left(\mathrm{cm}^{-1}\right)$ of compound VIb: $3450(\mathrm{OH}), 3250(\mathrm{NH}), 3050(\mathrm{CH}$ aromatic), 2950 (CH aliphatic), 1700 , $(\mathrm{C}=\mathrm{O}), 1680$ ( $\mathrm{NH}$ bending), 750 (C$\mathrm{Cl}) .{ }^{1} \mathrm{H} \quad \mathrm{NMR} \quad\left(\mathrm{DMSO}-\mathrm{d}_{6}\right)$ of compound VIb: $5.01 \quad(\mathrm{~s}, \quad 2 \mathrm{H},-$ $\mathrm{COCH}_{2} \mathrm{O}-, \quad 7.3-8.18 \quad(\mathrm{~m}, \quad 12 \mathrm{H}$, aromatic), 8.44 (d, 1H, H C-6), 10.34 $\mathrm{s}, 1 \mathrm{H}, \mathrm{OH}$ disappeared by $\mathrm{D}_{2} \mathrm{O}$ ), $11.26(\mathrm{~s}, 1 \mathrm{H}, \mathrm{NH}$ disappeared by $\mathrm{D}_{2} \mathrm{O}$ ). MS $\mathrm{m} / \mathrm{z}$ (rel. aband. \%) of compound VIb: 488.6 (2.20), 404.8 (3.30), 329.2 (77.66), 298.0 (15.75), 256.0 (73.99), 178.0 (100.00), 139.95 (21.61), 76.0 (36.26).

\section{1-(4-Chlorophenyl)-4-[(4-oxo-5,5-} diphenylimidazolin-2-yl)oxymethylcarbonyl amino]imidazo[1,5-a] quinazolin-5(4H)-one VIII

A mixture of 4-chloromethylcarbonylamino-1-(4-chlorophenyl)imidazo[1,5-a]quinazo-lin-5(4H)-one V (0.01 mol; $3.87 \mathrm{~g})$ and phenytoin sodium $(0.01 \mathrm{~mol} 2.74 \mathrm{~g})$ in dimethyl formamide $(5 \mathrm{ml})$ was heated in a boiling water bath for 3 hours. The resulting solution was cooled, poured onto ice water. The solid product was filtered, washed with water, and crystallized from ethanol. M.P. $204^{\circ} \mathrm{C}$, Yield $83 \%$. Microanalysis of $\mathrm{C}_{33} \mathrm{H}_{23} \mathrm{ClN}_{6} \mathrm{O}_{4} \quad$ (603.05) calcd.: $\mathrm{C}$ 65.71, H 3.84, N 13.94. Found: C 65.20, H 4.30, N 13.70. UV [ $\lambda_{\text {max }}$. $(\log \varepsilon)]: 304.8$ (3.65). IR $\left(\mathrm{cm}^{-1}\right)$ : 3300-3200 (NH), $3050 \quad(\mathrm{CH}$ aromatic), 2900-2850 (CH aliphatic),
$1780(\mathrm{C}=\mathrm{O}), 1620$ (NH bending), 750 (C-Cl). ${ }^{1} \mathrm{H}$ NMR (DMSO-d 6 ): 4.40(s, $\left.2 \mathrm{H},-\mathrm{COCH}_{2} \mathrm{O}-\right), 7.30-7.94(\mathrm{~m}, 18 \mathrm{H}$, $\mathrm{CH}$ aromatic), 8.09 (d, 1H, $\underline{\mathrm{HC}}-6)$, $9.77\left(\mathrm{~s}, 1 \mathrm{H}, \mathrm{NH}\right.$ disappeared by $\left.\mathrm{D}_{2} \mathrm{O}\right)$,

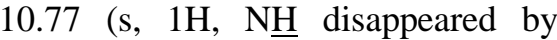
$\mathrm{D}_{2} \mathrm{O}$ ). MS m/z (rel. aband. \%): 603.1 (1.22), 576.9 (1.63), 523.6 (1.32), 446.5 (2.95), 369.2 (2.34), 327.2 (2.85), 257.3 (4.48), 198.0 (2.85), $81.2(100.00)$.

1-(4-Chlorophenyl)-4-(phthalimidomethylcarbonylamino)imidazo[1,5a]quinazolin-5(4H)-one IX

A mixture of 4-chloromethylcarbonylamino-1-(4-chlorophenyl)imidazo[1,5-a] quinazolin-5(4H)-one V (0.01 mol; $3.87 \mathrm{~g}$ ) and potassium phthalimide $(0.01 \mathrm{moI} ; 1.85 \mathrm{~g})$ in dimethylformamide $(5 \mathrm{ml})$ was heated in a boiling water bath for 3 hours. The resulting solution was cooled, poured onto ice water. The solid product was filtered, washed with water, and crystallized from ethanol. M.P. $266^{\circ} \mathrm{C}$, Yield. Microanalysis of $\mathrm{C}_{26} \mathrm{H}_{16} \mathrm{ClN}_{5} \mathrm{O}_{4}$ (497.91): Calcd: C 62.71, H 3.23, N 14.06. Found: C $63.10, \mathrm{H}$ 3.50, $\mathrm{N}$ 13.70. UV $\left[\lambda_{\text {max. }}\right.$. $(\log \varepsilon)]: 292.6$ (3.95), 236.8 (4.65). IR $\left(\mathrm{cm}^{-1}\right): \quad 3242 \quad(\mathrm{NH}), \quad 3061 \quad(\mathrm{CH}$ aromatic), 2925-2854 (CH aliphatic), 1726, $1650 \quad(\mathrm{C}=\mathrm{O}), \quad 1614(\mathrm{NH}$ bending), $750 \quad(\mathrm{C}-\mathrm{Cl}) .{ }^{1} \mathrm{H} \quad \mathrm{NMR}$ $\left(\mathrm{DMSO}_{-} \mathrm{d}_{6}\right): \quad 4.86 \quad$ (s, $2 \mathrm{H}$, $\left.\mathrm{NHCOCH}_{2}-\right), 7.22-7.94(\mathrm{~m}, \quad 12 \mathrm{H}$, aromatic), 8.07 (d, 1H, H C-6). MS m/z (rel. aband. \%): 497 (13.95), 466 (15.12), 356 (26.74), 342 (32.56), 263 (25.58), 211 (45.35), 160 (46.51),114 (100.00). 
4-(4-Chlorophenyl) imidazo[5,4,3-c] 1,2,3-triazolo [4,3-b] quinazolin$10(10 H)$-one XIII

To a solution of $\mathbf{I}(0.01 \mathrm{~mol} ; 3.1$ g) in $1 \mathrm{~N}$ hydrochloric acid $(20 \mathrm{ml})$ sodium nitrite solution $(10 \% ; 10 \mathrm{ml})$ was added while stirring in ice bath. The mixture was stirred for one hour. The mixture was boiled for 5 minutes, cooled, and extracted with methylene chloride $(3 \times 5 \mathrm{ml})$. The combined organic layer was collected, dried on anhydrous sodium sulphate. The excess solvent was removed under vacuum. The separated solid was crystallized from ethanol. M.P.: $242^{\circ} \mathrm{C}$, Yield $68 \%$. Microanalysis of $\mathrm{C}_{16} \mathrm{H}_{8} \mathrm{ClN}_{5} \mathrm{O}$ (321.75): Calcd.: C 59.72, H 2.50, N 21.76. Found: C 59.50, H 2.80, N 22.00.UV [ $\lambda_{\max .}(\log$ ع)]: 235.2(4.44). IR $\left(\mathrm{cm}^{-1}\right): 3050(\mathrm{CH}$ aromatic), $1680(\mathrm{C}=\mathrm{O}), 760(\mathrm{C}-\mathrm{Cl})$. ${ }^{1} \mathrm{H}$ NMR (DMSO- $\mathrm{d}_{6}$ ): 7.12-7.91 (m, $8 \mathrm{H}$, aromatic). MS m/z (rel. aband.\%): 323 (68.65), 321 (42.70), 285 (42.70), 211 (32.43), 162 (71.36), 132 (64.86), 115 (100.00), 62 (98.92).

\section{PHARMACOLOGICAL SCREENING}

\section{General behaviour of acute toxicity}

Mice of both sexes weighing 20$25 \mathrm{~g}$ were used to study the toxicological effect of the chosen compounds. Animals were observed within 24 hours for any mortality. It was found that all compounds are safe up to the highest chosen dose.

\section{Anti-inflammatory activity}

The anti-inflammatory activity of I and the new imidazo[1,5-a]quinazolin-5(4H)-one derivatives IIc, IIIa,
IIIf, IVa, V, VIc, VIIb, and XIII were tested using indomethacin as reference. The tested compounds and indomethacin were prepared as a suspension in $2 \%$ Tween 80 . The method of carrageenan- induced paw edema of Winter et al ${ }^{29 \& 30}$ was used to induce inflammation in this study. The percentage inhibition of inflammation was calculated according to the following equation:

$\%$ Inhibition $=$

Wt. of paw edema of control wt of paw edema of treated X 100

Wt. of paw edema of control

\section{Result and discussion}

Results are recorded in Table 3 and illustrated by Figures 1 and 2. It is obvious from the dose response curve that there is a direct relationship between the dose and the antiinflammatory activity. Compound I showed in a previous work ${ }^{11}$ higher anti-inflammatory than the reference drug flufenamic acid in a dose of 50 $\mathrm{mg} / \mathrm{kg}$. Replacement of the amino group by hydroxybenzylidene IIc, urea IIIa, thiourea IIIf, acetamide IVa, chloroacetamide $\mathbf{V}$ or substituted aminomethycarbonylamino VIc groups increases the activity than the parent amino derivative $\mathbf{I}$. The percentage inhibition of compounds IIc, IIIa, IIIf, IVa, V, and VIc in a dose of $50 \mathrm{mg} / \mathrm{kg}$ was $84.84,54.54$, $56.06,54.54,74.24,69.62$ respectively. The tetracyclic derivative XIII showed a marked decrease in the antiinflammatory activity than the tricyclic amino derivative $\mathbf{I}$. 
The most active compound was IIc that has 4-hydroxybenzylidene group. Its percentage inhibition was 84.84 in a dose of $50 \mathrm{mg} / \mathrm{kg}$.

The chloroacetyl derivative $\mathbf{V}$ was much more active than the acetyl derivative IVa where their percentage inhibition was 75.24 and 54.54 respectively.

The aminomethycarbonylamino derivative VIc was more active than the acetamide derivative IVa but less active than the chloroacetyl derivative and its percentage of inhibition was 69.62 in a dose of $200 \mathrm{mg} / \mathrm{kg}$.

Carbamide and thiocarbamide formation IIIa and IIIf slightly increases the anti-inflammatory activity than the parent amine $\mathbf{I}$, where their percentage inhibition was 54.54 and 56.05 respective.

The tetracyclic derivative XIII showed a marked decrease in the antiinflammatory activity than the tricyclic amine derivative $\mathbf{I}$ and its percentage inhibition was 24.24 in a dose of $200 \mathrm{mg} / \mathrm{kg}$. Moreover, esterification of compound $\mathbf{V}$ led to inactive derivative.

From the dose-response curves (Fig. 2), it is obvious that there is a direct relationship between the dose and the percentage inhibition.

Table 3: Effect of new imidazo[1,5-a]quinazolin-5(4H)-one derivatives, and indomethacin on carrageenan-induced paw edema in rats.

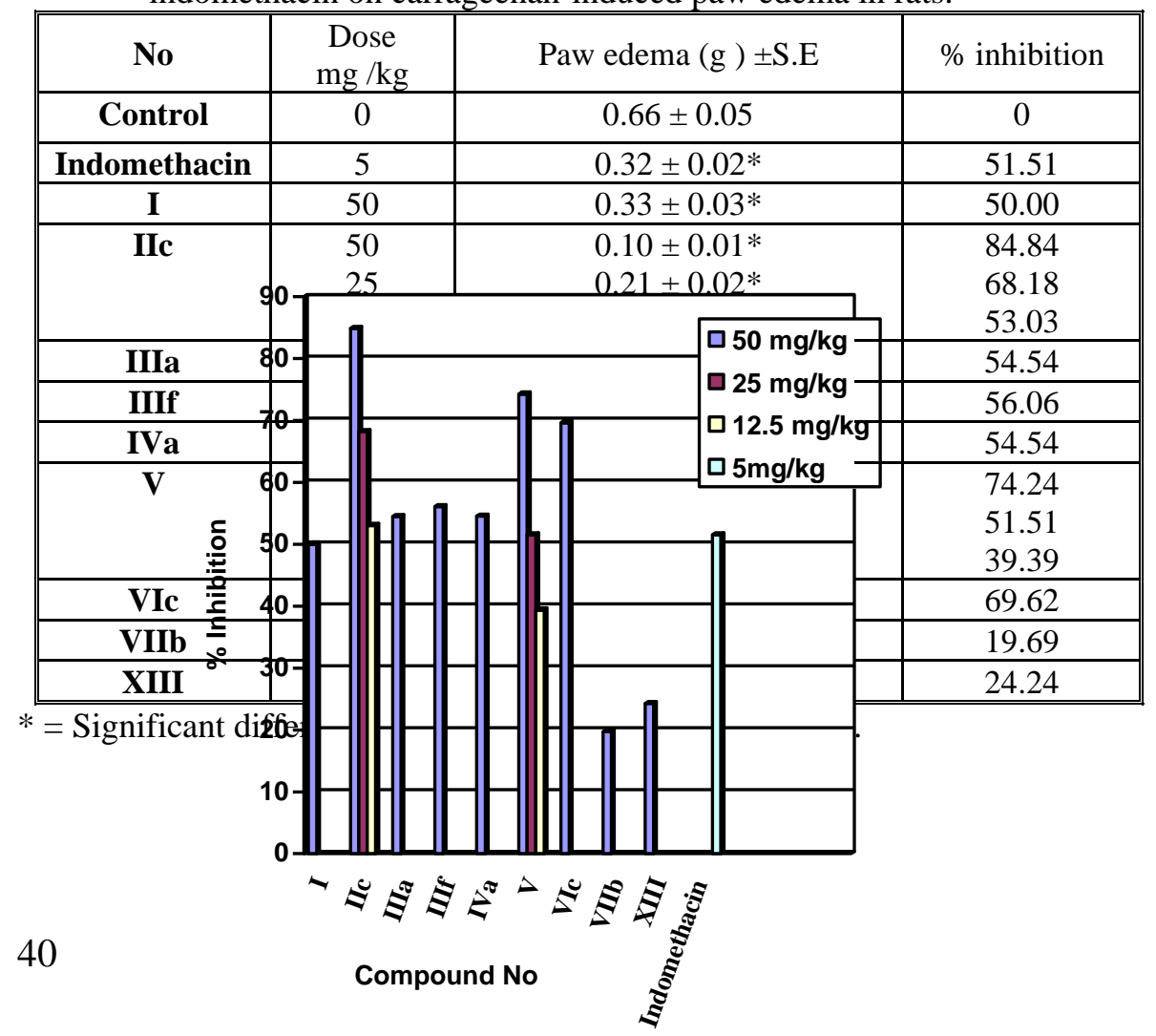




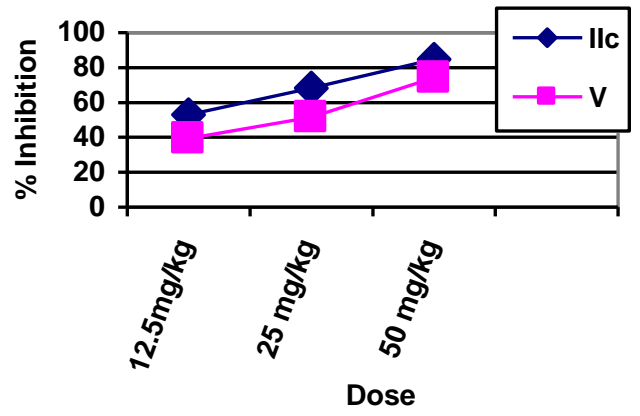

Fig. 2: Dose-response curve for compounds IIc and V.

\section{Analgesic activity}

Compounds showed higher antiinflammatory activity IIc, and $\mathbf{V}$ were chosen to study their analgetic activity.

Adult male albino mice weighing 20 - $25 \mathrm{~g}$ were used in this study. The new tested compounds and the reference drug indomethacin were prepared as a suspension in $2 \%$ Tween 80. The method of Okun et $\mathrm{al}^{31}$ was used to induce writhing in this study.

$\%$ Protection $=$

Number of protected animals x 100

Total number of animals

\section{Results and discussion}

Results are recorded in Table 4 and illustrated by Figure 3 . Compounds IIc and V in a dose of 25 $\mathrm{mg} / \mathrm{kg}$ showed higher analgesic activity than the reference drug indomethacin in a dose of $5 \mathrm{mg} / \mathrm{kg}$. The percentage protection of IIc, Vand indomethacin was 83.33, 100.00, and 66.66 respectively. Moreover, compound VI was proved to be equipotent to the reference drug in a dose of $12.5 \mathrm{mg} / \mathrm{kg}$.

Table 4: Analgesic activity of new imidazo[1,5-a] quinazolin-5(4H)-one derivatives and indomethacin using p-benzoquinone induced writhing in mice. 


\begin{tabular}{||c|c|c|c|c||}
\hline No & $\begin{array}{c}\text { Dose } \\
\mathrm{mg} / \mathrm{kg}\end{array}$ & $\begin{array}{c}\text { No. of } \\
\text { animals }\end{array}$ & $\begin{array}{c}\text { No of protected } \\
\text { animals }\end{array}$ & $\%$ protection \\
\hline Control & 0 & 6 & 0 & 0 \\
\hline Indomethacin & 5 & 6 & 4 & 66.66 \\
\hline IIc & 25 & 6 & 5 & 83.33 \\
& 12.5 & 6 & 3 & 50.00 \\
\hline $\mathbf{V}$ & 25 & 6 & 6 & 100.00 \\
& 12.5 & 6 & 4 & 66.66 \\
\hline
\end{tabular}

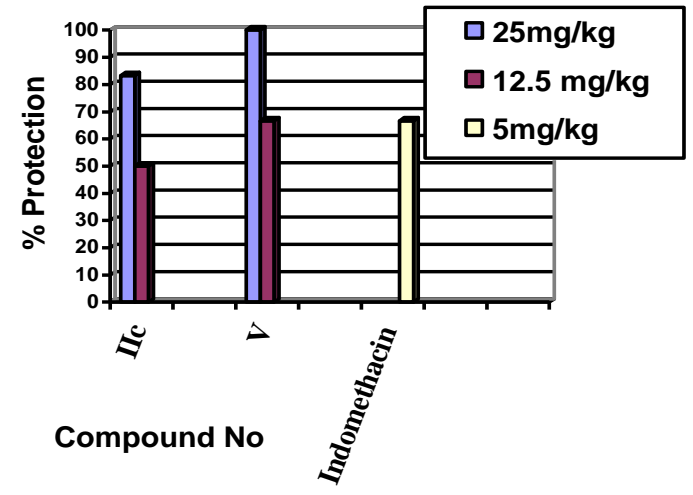

Fig. 3: Analgesic activity of imidazo[1,5-a] quinazolin-5(4H)-one derivatives and indomethacin using p-benzoquinone induced writhing method.

\section{Antipyretic activity}

Compounds which were tested for their analgesic activity IIc, and Vwere also chosen to study their antipyretic activity. Male albino mice weighing 20-25 $\mathrm{g}$ were chosen for this study according to Loux et al method $^{32}$. Rectal body temperature of the animals was measured after one and two hours from drug administration.

\section{Results and discussion}

Results are recorded in Table 5 and illustrated by Figure 4 . Compound $\mathbf{V}$ was more active as antipyretic than compound IIc in a dose of $50 \mathrm{mg} / \mathrm{kg}$ after two hours of drug administration (the difference in body temperature was 1.54 and $1.36^{\circ} \mathrm{C}$ respectively).

Table 5: Effect of imidazo[1,5-a]quinazolin-5(4H)-one derivatives and indomethacin on yeast induced hyperthermia in mice.

\begin{tabular}{|c|c|c|c|c|}
\hline \multirow[b]{2}{*}{ No } & \multirow[b]{2}{*}{$\begin{array}{c}\text { Dose } \\
\mathrm{mg} / \mathrm{kg}\end{array}$} & \multicolumn{3}{|c|}{ Average rectal body temperature ${ }^{\circ} \mathrm{C} \pm$ S.E. } \\
\hline & & $\begin{array}{c}\text { Pre- } \\
\text { administration }\end{array}$ & $\begin{array}{l}\text { One hour post } \\
\text { administration }\end{array}$ & $\begin{array}{l}\text { Two hours post } \\
\text { administration }\end{array}$ \\
\hline Control & 0 & $38.28 \pm 0.21$ & $38.25 \pm 0.17$ & $38.36 \pm 0.17$ \\
\hline
\end{tabular}




\begin{tabular}{|c|c|c|c|c||}
\hline Indomethacin & 5 & $38.26 \pm 0.18$ & $36.72^{*} \pm 0.22$ & $36.58^{*} \pm 0.20$ \\
\hline IIc & 25 & $38.13 \pm 0.25$ & $37.76 \pm 0.29$ & $37.58 \pm 0.55$ \\
& 50 & $38.10 \pm 0.16$ & $36.46^{*} \pm 0.49$ & $36.74^{*} \pm 0.40$ \\
\hline V & 25 & $37.94 \pm 0.07$ & $37.92 \pm 0.24$ & $37.18^{*} \pm 0.25$ \\
& 50 & $38.02 \pm 0.21$ & $36.54^{*} \pm 0.17$ & $36.60^{*} \pm 0.18$ \\
\hline
\end{tabular}

$*=$ Significant difference from the control value at $\mathrm{p}<0.05$

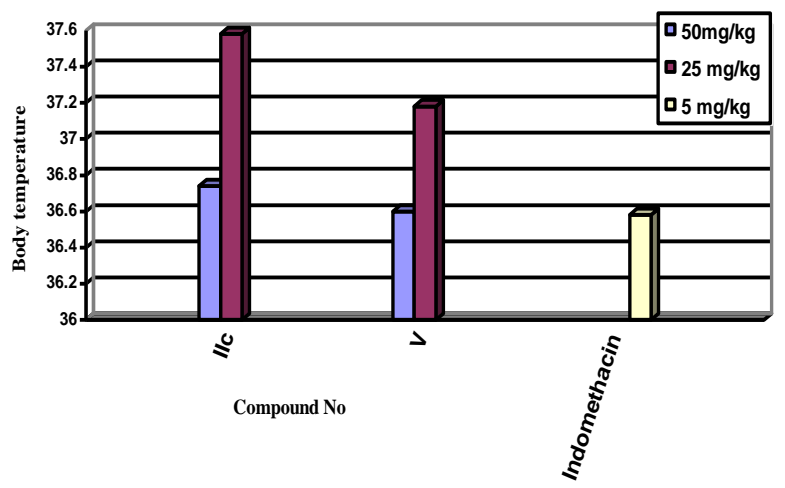

Fig. 4: Antipyretic activity of imidazo[1,5-a] quinazolin-5(4H)-one derivatives and indomethacin.

\section{Ulcerogenic effect}

Compounds IIc and V were subjected to further study for their ulcerogenic effect. Adult male albino rats weighing $120-150 \mathrm{~g}$ were used in this study. Animals were fasted eighteen hours before the drug administration $^{33}$. The ulcer index was calculated according to the method of Robert et $\mathrm{al}^{34}$. The degree of ulcerogenic effect was expressed in term of:

I- Percentage incidence of ulcers in each group of animals divided by 10

II- The average number of ulcers per stomach.
III- The average severity of ulcers by visual observation.

The ulcer index is the value that result from the sum of the above three values.

\section{Results and discussion}

Results are recorded in Table 6 and illustrated by Figure 5. Result revealed that indomethacin in a dose of $5 \mathrm{mg} / \mathrm{kg}$ showed an ulcer index of 17.60. Both compounds IIc and V in a dose of $50 \mathrm{mg} / \mathrm{kg}$ showed slight decrease in their ulcer indices than the indomethacin. Their ulcer indices were 17.14 and 16.25 respectively.

Table 6: Ulcerogenic effect of the new anthranilate analogs, imidazo[1,5-a] quinazolin-5(4H)-one derivatives, flufenamic acid, and indomethacin. 


\begin{tabular}{||c|c|c|c|c|c|c||}
\hline No & $\begin{array}{c}\text { Dose } \\
\mathrm{mg} / \mathrm{kg}\end{array}$ & $\begin{array}{c}\text { Rats } \\
\text { No }\end{array}$ & $\begin{array}{c}\text { \% Incidence } \\
\text { divided by 10 }\end{array}$ & $\begin{array}{c}\text { Average No } \\
\text { of ulcer }\end{array}$ & $\begin{array}{c}\text { Average } \\
\text { severity }\end{array}$ & $\begin{array}{c}\text { Ulcer } \\
\text { index }\end{array}$ \\
\hline Control & 0 & 5 & 0 & 0 & 0 & 0 \\
\hline Indomethacin & 5 & 5 & 10 & 6.0 & 1.60 & 17.60 \\
\hline IIc & 50 & 5 & 10 & 5.6 & 1.54 & 17.14 \\
\hline V & 50 & 5 & 10 & 4.8 & 1.45 & 16.25 \\
\hline
\end{tabular}

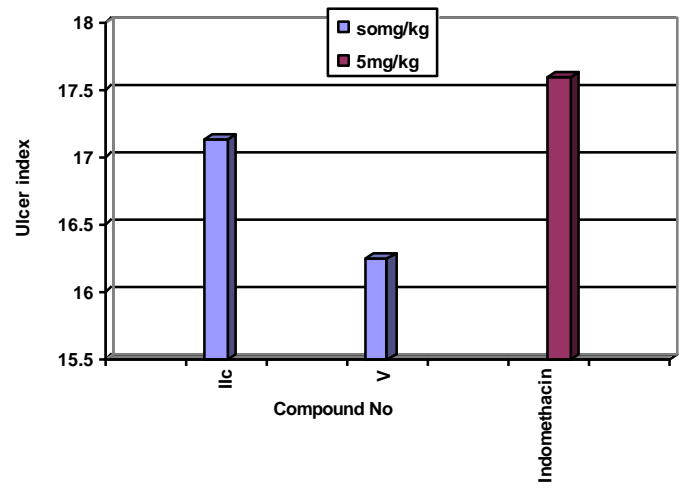

Fig. 5: Ulcerogenic effect imidazo[1,5-a] quinazolin-5(4H)-one derivatives and

\section{Anticonvulsant activity}

Compounds I, IIIc, VIc and VIII which contain ureido or hydantoin function were chosen for this study using diazepam as a reference drug. Mice of both sexes weighing 20-25 g were used for this study. Animals were stimulated through ear electrode of $50 \mathrm{~mA}$ as a single stimulator for 0.2 sec. $^{35 \& 36}$. The anticonvulsant activity was expressed as the percentage protection according to the following equation:

$\%$ Protection $=$

Number of protected animals x 100

Total number of animals

\section{Results and discussion}

Results were recorded in Table 7, and illustrated by Figure 6. All the chosen compounds I, IIIc, VIc, and VIII exhibited anticonvulsant activity and their PD $_{50}$ were 50, 25, 25, and $50 \mathrm{mg} / \mathrm{kg}$ respectively. Replacement of the amino group of I by ureido IIIc or aminomethycarbonylamino VIc led to increase in the anticonvulsant activity. The phenytoin derivative VIII did not change the efficacy of the parent amine $\mathbf{I}$. 
Table 7: Anticonvulsant activity of imidazo[1,5-a]quinazolin-5(4H)-one derivatives and diazepam.

\begin{tabular}{||c|c|c|c||}
\hline No & $\begin{array}{c}\text { Dose } \\
\mathrm{mg} / \mathrm{kg}\end{array}$ & $\begin{array}{c}\text { No of animals did } \\
\text { not convulse }\end{array}$ & $\%$ Protection \\
\hline Control & 0 & $0 / 6$ & 0 \\
\hline Diazepam & 5 & $3 / 6$ & 50.00 \\
\hline I & 25 & $2 / 6$ & 33.33 \\
& 50 & $3 / 6$ & 50.00 \\
\hline IIc & 25 & $3 / 6$ & 50.00 \\
& 50 & $5 / 6$ & 83.33 \\
\hline Vic & 25 & $3 / 6$ & 50.00 \\
& 50 & $5 / 6$ & 83.33 \\
\hline VIII & 25 & $2 / 6$ & 33.33 \\
& 50 & $3 / 6$ & 50.00 \\
\hline
\end{tabular}

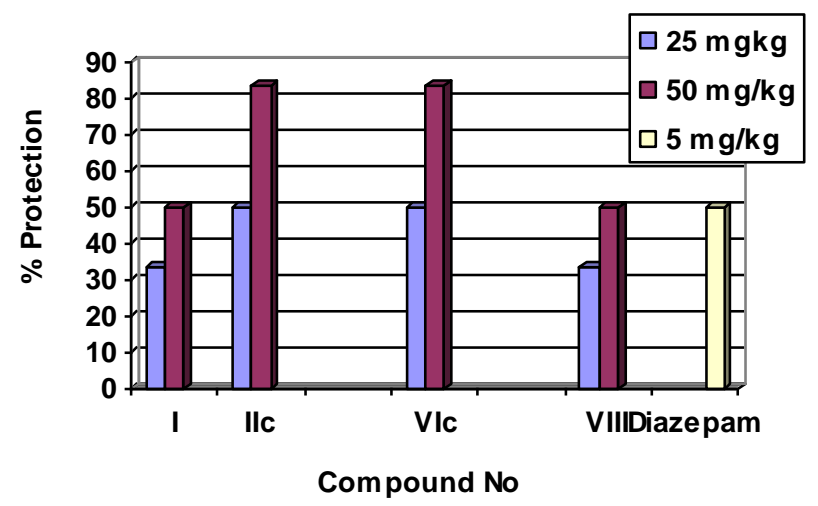

Fig. 6: Anticonvulsant activity of imidazo[1,5-a]quinazolin-5(4H)-one derivatives and diazepam.

REFERENCES
1- J. W.Chern, C. Y. Shiau and G. y. Lu, Bioorg. Med. Chem. Lett., 1, $571(1991)$. 
2- J. M. Yang, T. C. Yuen, C.W. Chang, J. S. Jing, M. H.Yen and J. W. Chern, J. Cardiovasc. Pharmacol., 30, 229, (1997). Through C.A. 128, 225918s (1998).

3- Y. T. Huang, H. L. Wu, J. W. Chern, H.C. Lin and C. Y. Hong, Scand. J. Gastroenterol., 33, 1303 (1998).

4- J. W. Chern, P. L. Tao, K. C. Wang, A. Gutcait, S. W. Liu, M. H. Yen, S. L. Chien and J. K. Rong, J. Med. Chem., 41, 3128 (1998).

5- D. De Chaffoy De Courcelles D., K. De loore, E. Freyne and P. A. J. Janssen, J. Pharm. Exp. Ther., 263, 6, (1992).

6- Q. Li, M. M. Himmel and U. Ravens, J. Cardiovasc. Pharmacol., 24, 133 (1994).

7- G. E. Hardtmann, G. Koletar, O. R. Phister, J. H. Gogerty and L. C.Iorio, J. Med. Chem., 18, 447 (1975).

8- N. P. Peet and S. Sunder, U.S. US 4，871，732(Cl. 514-212; A61k31/505), 03 Oct. 1989, Appl. 247, 797, 22 Sep. 1988, 6pp. Through C.A. 112, 179036v (1990).

9- G. W. Rewcastle, B. D.Palmer, A. J. M. Bridges, H. D. H. Showalter, L. Sun, J. M. A. Nelson, A. J. Kraker, B. W. Fry and W. A. Penny, J. Med. Chem., 39, 918 (1996).

10- G. Wagner and E. Bunk, Pharmazie, 34, 209 (1979).

11- M. M. El-kerdawy, M. B. ElAshmawy, I. A. Shehata, A. E. M. Barghash, E. R. El-Bendary and H. A. El-kashef, Saudi. Pharm. J., 5, 46 (1997).

12- V. R. Ztets, R. S. Sinyak and I. A. Mazur, Fram Zh., 3, 40 (1984). Through C.A. 101, 183449z (1984).

13- S. Inaba and H. Yamamoto, U.S. US 3, 891, 638 (Cl. 260-244R; C07d), 24 Jan. 1975, Appl. 172, 562, 17 Aug. 1971, 7pp. Through C. A. 84, 17406u (1976).

14- R. E. Rodway and R. F. Cookson, S. African 72 01, 118(Cl. C07d), 21 Aug. 1973, Appl. 72/ 1118, 21 Feb. 1972; 138pp. Through C. A. 81, 13559m (1974).

15- Sumitomo Chemical Co., ltd., Jpn. Kokai Tokkyo Koho. 80, 55, 188 (Cl. 07D 487/04), 22 Apr. 1980, Appl. 78/129, 811, 20 Oct. 1978, 5pp. Through C.A. 94, 65713v (1981).

16- M. Yamamoto, M. Koshiba and H. Yamamoto, Ger. Offen 2, 805, 124(Cl. CO7D 487/04), 10 Aug. 1978, Japan Appl.77, 13, 818, 09 Feb. 1977; 29pp. Through C. A. 89, 197593n (1978).

17- G. E. Hardtmann, U.S. 4, 042, 511(Cl. 260-244A, C07D 265/100), 24 May 1977, Appl. 536, 099, 24 Dec. 1974, 4pp. Through C. A. $85,85044 \mathrm{~m}$ (1977).

18- W. Optiz, H. Jacobi and B. Pelster, Ger. Offen DE 3, 220, 438(Cl. C 07D 487/04), 01 Dec. 1983, Appl.29 May 1982; 27pp. Through C. A. 100, 103383q (1984).

19- G. E. Hardtmann, U.S. 4, 013, 646(Cl. 260-244A, C 07D 
265/26), 22 Mar. 1977, Appl. 373, 474, 25 Jun 1973; 6pp. Through C. A. $87, \quad 23325 \mathrm{v}$ (1977).

20- G. E. Hardtmann G. E., U.S. 3, 894, 022(Cl. 260-256.4F; C07D), 08 July 1975, Appl. 374, 474, 27 Jun 1973, 6pp. Through C. A. 83, 164225s (1975).

21- H. C. Jackson, H. C. Hansen, M. Kristiansen, P. D. Suzadak, M. E. Judge and M. D. B. Swedberg, Br. J. Pharmacol., 114, 288p (1995).

22- H. C. Jackson, H. C. Hansen, M. Kristiansen, P. D. Suzadak, H. Klitgaad, M. E. Judge and M. D. B. Swedberg, Eur J. Pharmacol., 308, 21 (1996).

23- W. öscher, C. Rundfeldt, D. Hönack and U. Ebert, J. Pharm. Exp. Ther., 279, 573 (1996).

24- $\mathrm{H}$. C. Hansen and M. Kristiansen, PCT Int Appl. WO 93 13, 103(Cl. C 07D 487/04), 08 Jul. 1993, DK Appl. 91/2, 042, 20 Dec. 1991, 4pp. Through C. A. 119 24996q (1993).

25- H. C. Ansen, PCT Int Appl. WO 92 00, 298(Cl. C 07D 487/04), 09 Jan. 1992, DK Appl. 90/1, 518, 22 Jun 1990, 19pp. Through C. A. 116, p174168q (1992).

26- F. Watjen and H. C. Hansen, Eur. Pat. Appl. EP 283, 162(Cl. C 07D 487/04), 21 Sep. 1988, DK Appl. 87/1, 374, 18 Mars 1987; 19 pp. Through C.A. 110, 57685w (1989).

27- H. H. Hassanein, F. A. Ragab, E. I. Aly and H. H. Georgy, Alex. J. Pharm. Sci., 20, 129 (2006).

28- H. H. Hassanein, Zogazig J. Pharm. Sci., 3, 172 (1994).
29- C. A. Winter, E. A. Risley and G. W. Nuss, J. Pharm. Exp. Ther., 141, 369 (1963).

30- C. A. Winter, E. A. Risley and G. W. Nuss., Proc. Soc. Exp. Biol. Med., 111, 544 (1962).

31- R. Okun, S. C. Liddon and L. Lasagna, J. Pharm. Exp. Ther., 139, 107 (1963).

32- J. Loux, P. Depalma and S. Yankell, Toxicol. Appl. Pharmacol., 22, 672 (1972). 
33- M. Meshali, E. El-Sabbah and A. Foda, Acta Pharm. Technol., 29, 217p (1983).

34- A. Robert, E. W. Negamis and J. P. Philips, Gastroenterology, 55, 481 (1968).

35- L. A. Woodgurg and V. D. Davenport, Arch. Int. Pharmacodyn., 92, 97 (1952).

36- H. G. Vogel and W. H. Vogel, "Drug Discovery and Evaluation", Pharmacological Assay, Spring-Verlag Berlin, Herdelberg, 1997, p. 260. 